\title{
Velocidade de Processamento da Informação na Definição e Avaliação da Inteligência
}

\author{
Iolanda S. Ribeiro ${ }^{1}$ \\ Leandro S. Almeida \\ Universidade do Minho
}

\begin{abstract}
RESUMO - A relação entre os tempos de reação em tarefas simples e complexas, ou os tempos de inspeção, e os resultados em testes de inteligência tem sido analisada ao longo dos tempos. Neste artigo descrevem-se os procedimentos usados na avaliação dos tempos de reação e tempos de inspeção, apresentam-se os coeficientes de correlação que tais tarefas mantêm com os testes de inteligência, assim como algumas das explicações avançadas pelos autores para os dados obtidos. Mesmo que os índices de correlação oscilem em função da natureza das tarefas, e sobretudo do grau de homogeneidade ou heterogeneidade das amostras avaliadas, os estudos apontam, de uma forma sistemática, para correlações negativas e estatisticamente significativas. No entanto, o grande desafio para esta linha de investigação continua a ser a construção e testagem de uma teoria psicológica explicativa dos coeficientes obtidos.
\end{abstract}

Palavras-chave: inteligência; processamento de informação; memória a curto prazo.

\section{Speed of Information Processing on Intelligence Definition and Assessment}

\begin{abstract}
The relationship between the reaction time in simple or complex tasks, or the inspection time, and the results in intelligence tests has been analysed over time. In this article, the procedures used in the assessment of both reaction and inspection time are described. Correlation coefficients that such tasks hold with intelligence tests are also presented, as well as some explanations given by the authors. Even though the correlation indexes vary according to the nature of the tasks, and namely with the degree of subjects homogeneity or heterogeneity in the samples, studies systematically point to negative and statistically significant correlations. Nevertheless, the most important challenge in this research field is to develop and to test a psychological theory that explains the correlations found.
\end{abstract}

Key words: intelligence; information processing; short-term memory.

A relação entre a velocidade com que são executadas tarefas cognitivas simples e a inteligência aparece estudada desde os primórdios da Psicologia com os trabalhos de Galton e Cattell (Bates \& Stough, 1998). No entanto, as baixas correlações obtidas entre rendimento acadêmico e os tempos de reação (Sharp, 1898/99; Wissler, 1901) condicionaram, de forma decisiva, a atitude e o interesse dos psicólogos por este tipo de estudos. Este interesse ressurgiu na década de cinqüenta do século passado, assumindo-se que a velocidade de processamento de informação constitui um componente básico das diferenças individuais na inteligência (Fink \& Neubauer, 2001, 2005; Rinderman \& Neubauer, 2004). Os trabalhos desenvolvidos por Hick (1952) e Hyman (1953) são dos primeiros que se situam nesta linha. A metodologia seguida, desde então, consiste na avaliação dos sujeitos em medidas de inteligência geral e em tarefas cognitivas elementares (Smith \& Stanley, 1983) e no cálculo da correlação entre as duas variáveis.

Neste artigo, de uma forma necessariamente breve, procuramos sistematizar a informação relativa à investigação nesta área. Mais concretamente procuramos responder a duas

1 Endereço: Universidade do Minho, Instituto de Educação e Psicologia, Campus de Gualtar, Braga, Portugal 4710-057. E-mail: iolanda@ iep. uminho.pt questões, que nos parecem centrais, sobre a relação entre a velocidade de processamento da informação e a inteligência: (i) o que sabemos e (ii) o que desconhecemos.

\section{Velocidade de processamento da informação e inteligência: o que sabemos}

No estudo da velocidade de processamento de informação têm sido utilizadas diferentes tarefas diferenciadas entre si no volume e natureza da informação a processar, nomeadamente se apela ou não à informação retida na memória a curto prazo. No paradigma de Hick (1952), avalia-se o tempo de reação a estímulos auditivos ou visuais em função da quantidade de informação transmitida. São calculados tempos de reação simples ou com duas escolhas, envolvendo 0 e 1 unidades (bits) de informação respectivamente (Jensen, 1979a). A resolução destas tarefas não requer o acesso à memória a curto ou a longo prazo, sendo o tempo requerido para processar os estímulos uma função da informação neles presente. Este procedimento foi aprimorado por Jensen (1979b), que aumentou o volume de informação a processar. Para tal recorreu a painéis com uma, duas, quatro ou oito combinações de luzes e botões, o que permitia variar o número de alternativas de escolha dos sujeitos e aumentar as unidades de informação a processar. 
No seu conjunto, os resultados das investigações têm mostrado, de forma consistente, a existência de correlações negativas e estatisticamente significativas entre os parâmetros derivados de tarefas simples de tempos de reação e os resultados em provas de inteligência geral (Jensen, 1979b; Jensen \& Munro, 1979; Deary, Der \& Ford, 2001). Estes resultados foram confirmados em diferentes amostras: crianças normais (Jensen \& Munro, 1979), adolescentes superdotados (Cohn, Carlson \& Jensen, 1985), crianças com atraso mental severo (Jensen, Schafer \& Crinella, 1981) ou com atraso moderado (Vernon, 1981), trabalhadores manuais não qualificados (Sen, Jensen, Sen \& Arora, 1983), alunos universitários (Vernon, 1983a, 1983b) ou adultos (Barrett, Eysenck \& Lucking, 1986). Mais recentemente, Deary e cols. (2001) verificaram que a magnitude do efeito não se alterava quando se considera o sexo, a classe social e o nível de instrução escolar dos sujeitos.

Ainda de forma consistente, observam-se variações nos valores das correlações em função da complexidade das tarefas. Os coeficientes são mais elevados quando os tempos de reação são calculados recorrendo a tarefas mais complexas (Levine, Preddy \& Thorndike, 1987; Lindley, Wilson, Smith \& Bathurst 1995; Necka, 1992; Vernon \& Weese, 1993). A relação entre a complexidade das tarefas e o QI não é, no entanto, linear (Jensen, 1993), apontando os dados para a existência de um nível ótimo de complexidade. Nas tarefas muito simples, os tempos de reação refletiriam mais processos sensório-motores do que processos cognitivos de tratamento de informação; por sua vez, nas tarefas muito complexas emergiriam outros fatores, por exemplo, estratégias metacognitivas, a influenciar a velocidade de processamento da informação (Lindley \& cols., 1995). As correlações mais elevadas serão obtidas quando as tarefas de tempos de reação se aproximam do limiar da memória de trabalho, ou seja, quando implicam uma quase sobrecarga da memória de trabalho. Quando essa capacidade é ultrapassada, registra-se uma redução nos coeficientes de correlação.

Embora reconhecendo a existência de correlações entre as duas variáveis, algumas objeções foram levantadas ao nível dos procedimentos usados, os quais podiam influenciar a magnitude das correlações observadas. Por exemplo, sugeria-se que tais coeficientes oscilavam consoante a aplicação dos testes de inteligência era feita com ou sem limites de tempo (Carroll, 1981; Schwartz, Griffin \& Brown, 1983; Vigneau, Blanchet, Loranger \& Pépin, 2002) ou consoante se controlava ou não o efeito da prática acumulada ao longo do experimento (Longstreth, 1984; Longstreth, Walsh, Alcorn \& Szzeeszulski, 1986). Respondendo a estas críticas, Vernon, Nador e Kantor (1985) verificaram que as correlações se mantinham independentemente das condições (com e sem limites de tempo) em que eram aplicados os testes de inteligência. No entanto, o efeito da prática acumulada permanece por esclarecer. Neubauer (1991), após eliminar o efeito identificado por Longstreth, verificou um aumento nas correlações entre tempos de reação e QI. Também Larson e Saccuzzo (1986), controlando o efeito de prática, não confirmaram a hipótese de Longstreth. Porém, resultados divergentes foram apresentados em outros estudos (Boris, MacLeod \& Forrin, 1993).
Para além dos estudos centrados nos tempos de reação, algumas investigações recorrem a tarefas que requerem o acesso à informação retida na memória a curto prazo. Numa delas, começa-se por apresentar ao sujeito um pequeno número de dígitos (números ou letras) seguido imediatamente por um dígito-prova. Consoante este esteja, ou não, incluído no conjunto inicial de dígitos, o sujeito deve responder com "sim" ou "não". O tempo que o sujeito demora a carregar no botão correspondente a cada resposta (tempo de decisão) é tomado como um indicador da velocidade de procura na memória a curto prazo (Sternberg, 1966). De forma paralela ao paradigma de Hick, também aqui o tempo de reação aumenta em função do número de dígitos incluídos no conjunto (Jensen, 1979b), contudo nestas últimas tarefas experimentais o tempo de reação envolve o tempo de inspeção e o tempo de resposta propriamente dito.

Duas tarefas permitem-nos exemplificar a investigação sobre os tempos de inspeção. Vickers e colaboradores (Smith, 1986; Vickers \& Smith, 1986) apresentavam estímulos contendo duas linhas verticais diferentes ou iguais no seu comprimento. A visualização dos estímulos era seguida de uma máscara composta por duas linhas verticais com o mesmo comprimento, impedindo-se deste modo que o sujeito continuasse a visualizar o estímulo inicial. A tarefa do sujeito, em cada ensaio, consistia em indicar se o comprimento das duas linhas era igual ou diferente. Numa outra tarefa alterava-se a ordem em que acendiam oito lâmpadas dispostas em fila (Nettelbeck \& Kirby, 1983a). Depois de todas acesas, pedia-se ao sujeito que indicasse as que o foram em primeiro lugar

As correlações entre tempos de inspeção e resultados em testes de inteligência mostram-se consistentes nos diferentes estudos, contudo apresentam grande dispersão. Longstreth e cols. (1986) verificaram que os coeficientes de correlação oscilavam entre $-0,92$ e $-0,20$, contribuindo para essa variabilidade as características das amostras utilizadas. Correlações mais elevadas obtinham-se em amostras mais heterogêneas, por exemplo, incluindo deficientes mentais (Irwin, 1984; Longstreth \& cols., 1986; Vernon, 1986). A homogeneidade das amostras, por sua vez, reduzia os coeficientes de correlação obtidos (Crawford, Deary, Allan \& Gustaffson, 1998; Irwin, 1984; Vernon, 1983a, 1983b). A título exemplificativo, Nettelbeck (1982) encontrou correlações da ordem de -0,13 e - 0,34 em dois grupos de sujeitos cujos QI variavam entre 112-135 e entre 104-135, respectivamente.

Numa meta-análise das investigações centradas na relação entre inteligência e tempos de inspeção, Kranzler e Jensen (1989) verificaram que as correlações corrigidas se situavam à volta de $-0,54$ para adultos normais e $-0,47$ para crianças. Mais recentemente, Grudnik e Kranzler (2001) replicaram o estudo anterior com um maior número de investigações $(90$ versus 31 ) e de sujeitos (4100 versus 1120 ). Os dados obtidos confirmam de novo uma relação substancial entre as duas variáveis, sendo o valor da correlação de $-0,51$. Este estudo confirma que os índices de correlação oscilam em função da maior homogeneidade ou da maior heterogeneidade das amostras tomadas, e acrescenta o impacto da utilização pelos sujeitos de estratégias cognitivas específicas. Nos estudos que consideram esta variável, as correlações entre tempo de 
inspeção e inteligência são mais elevadas em sujeitos que verbalizam ter utilizado estratégias cognitivas mais apropriadas (Brand \& Deary, 1982; Hulme \& Turnbull, 1983; Nettelbeck \& Kirby, 1977, 1983b; Smith, 1986; Smith \& Stanley, 1983).

\section{Velocidade de processamento da informação e inteligência: o que desconhecemos}

A existência de associações entre medidas cognitivas de nível inferior (representada pelas tarefas de tempos de reação) e de nível superior (presentes nos testes de inteligência) é atualmente aceita (Ceci, 1990), a questão está em saber como isso se explica. A teoria da capacidade limitada da memória a curto prazo é uma das explicações disponíveis. As tarefas cognitivas complexas requerem que diferentes unidades de informação sejam retidas na memória a curto prazo para serem processadas no momento adequado. Esta informação não pode ser retida durante muito tempo sem ensaio, o qual pode não ser possível quando o sistema tem de executar outras tarefas em simultâneo (Necka, 1992). Aceitando que as tarefas cognitivas são executadas num subsistema da memória a curto prazo, ou seja a memória de trabalho, a velocidade com que são executadas pode constituir, então, um fator decisivo para explicar as diferenças de realização dos sujeitos (Vernon, 1983a, 1983b). É de esperar que, quanto mais rapidamente forem recuperadas e processadas as informações relevantes para a resolução de um problema, maior é a probabilidade do limiar de capacidade do sistema não ser ultrapassado. Do mesmo modo, na ausência de ensaio a informação na memória de trabalho está sujeita a um declínio ou desaparecimento rápidos. Daí que, numa situação de processamento lento, a informação codificada previamente ou recuperada pode ser perdida, ou a sua manutenção pode ser feita à custa da sobrecarga do sistema que, assim, se revela incapaz de executar os processos necessários à resolução de um problema. Dir-se-ia que, a cada momento, existe uma trade-off entre a informação que pode ser mantida na memória de trabalho e as operações que podem ser executadas sobre essa informação. Quanto maior for a quantidade de informação requerida por uma tarefa e quanto mais exigente for o processamento necessário, maior é a probabilidade de se verificar uma perda da informação codificada inicialmente. A velocidade com que é recuperada e processada a informação associa-se, assim, à probabilidade do sistema ficar sobrecarregado pelas necessidades, simultâneas, de retenção e de processamento.

Estas características da memória de trabalho explicam que a velocidade e eficiência com que são executados os componentes de uma tarefa se revelem fundamentais na resolução quer de tarefas simples de discriminação sensorial, quer dos testes de inteligência. De acordo com Necka (1992), a velocidade de processamento poderia ser perspectivada como uma condição, ou mesmo um pré-requisito da inteligência, constituindo-se como a melhor forma de ultrapassar a capacidade limitada do sistema de memória. Claro está que, apesar de esta conclusão se apresentar como lógica, é necessária alguma prudência na sua aceitação. Desde logo, é questionável que os tempos de reação sejam uma medida fidedigna da velocidade de processamento da informação. Por outro, as estratégias cognitivas e metacognitivas utilizadas pelos sujeitos interferem nas medidas obtidas e, inclusive, podem ser uma outra forma de agilizar a memória de trabalho nas necessidades, simultâneas, de retenção e processamento da informação (Fink \& Neubauer, 2005; Necka, 1992). Estas diferenças individuais, que não podemos assumir como totalmente independentes das suas aprendizagens e das experiências culturais, poderão modelar o funcionamento daquilo que vem sendo definido por "executivo central" ou estrutura de controle da atenção que asseguraria um fluxo organizado da informação na memória de trabalho (Baddeley, 1996; Hunt, 1999).

Importa, assim, reconhecer que uma explicação cabal e definitiva para as correlações encontradas entre os tempos de reação ou de inspeção e os resultados nos testes de inteligência continua ausente. Nettelbeck (2001), numa base que reconhece ser ainda especulativa, sugere algumas hipóteses que poderão vir a guiar a investigação futura. Contrariando a perspectiva generalizada que classifica os tempos de reação e os tempos de inspeção como "tarefas simples", lembra que a acuidade sensorial, a atenção, a percepção, as aptidões espaciais e de visualização ou os processos de tomada de decisão estão presentes na realização cognitiva de tais tarefas, podendo mediar também os desempenhos nos testes de inteligência.

Sobretudo é questionável o sentido linear que se tem dado às correlações obtidas entre tempos de reação/inspeção e resultados em testes de inteligências (Der \& Deary, 2003). Os modelos de equações estruturais têm sido cada vez mais utilizados, para compreender qual a posição das tarefas de velocidade de processamento de informação nos modelos hierárquicos da inteligência humana (Burns \& Nettelbeck, 2003), bem como a sua relação com a inteligência fluida, com a inteligência cristalizada e com um fator geral de velocidade (Burns \& Nettelbeck, 2003; Fink \& Neubauer, 2001; O'Connor \& Burns, 2003; Osmon \& Jackson, 2002; Petrill, Luo, Thompson \& Detterman, 2001). Todos estes estudos confirmam a associação tradicionalmente encontrada, mantendo-se no entanto parcos na sua explicação. Assim sendo, parece-nos pouco razoável que se assumam os tempos de reação ou de inspeção como medidas válidas da inteligência, ou que se assumam tais medidas como mais fidedignas e livres de influência cultural (Eysenck, 1985).

Importaria, em nossa opinião, convergir nos estudos para uma diversificação das tarefas usadas nos experimentos de acordo com uma teoria da inteligência consolidada, por exemplo, o recurso à teoria dos três estratos de Cattell-Horn-Carroll (Carroll, 1993; Horn \& Noll, 1997), assim como diversificar sub-amostras de sujeitos, por exemplo, em função dos seus estágios de desenvolvimento cognitivo (Williams, Hultshch, Strauss, Hunter \& Tannock, 2005; Zhengkui, Jiannong \& Li, 2003), ou, ainda, tomar o substrato neuroquímico da cognição (Stough, Thompson, Bates \& Nathan, 2001), para se avançar decisivamente na explicação pela psicologia das correlações encontradas entre velocidade de processamento da informação e resultados nos testes de inteligência. Só entendendo o que significa "velocidade de processamento de informação", em termos psicológicos, podemos assumi-la como variável relevante no estudo da cognição e da inteligência. 


\section{S. Ribeiro e L. S. Almeida}

\section{Referências}

Baddeley, A. D. (1996). Exploring the central executive. Quarterly Journal of Experimental Psychology, 49(1), 5-28.

Barrett, P., Eysenck, H. J. \& Lucking, S. (1986). Reaction time and intelligence: A replicated study. Intelligence, 10(1), 9-40.

Bates, T. C. \& Stough, C. K. (1998). Improved reaction time method, information-processing speed, and intelligence. Intelligence, 26(1), 53-62.

Boris, D. A., MacLeod, C. M. \& Forrin, B. (1993). Eliminating the IQ-IT correlation by eliminating an experimental confound. Intelligence, 17(4), 475-500.

Brand, C. R. \&. Deary, I. J. (1982). Intelligence and "inspection time”. Em H. J. Eysenck (Org.), A model for intelligence. New York: Springer.

Burns, N. R. \& Nettelbeck, T. (2003). Inspection time in the structure of cognitive abilities: Where does it fit?. Intelligence, 31(3), 237-255.

Carroll, J. B. (1981). Ability and task difficulty in cognitive psychology. Educational Research, 10(1), 11-21.

Carroll, J. B. (1993). Human cognitive abilities: A survey offactoranalytic studies. New York: Cambridge University Press.

Ceci, S. J. (1990). On the relation between microlevel processing efficiency and macrolevel measures of intelligence. Intelligence, 14(2), 141-150.

Cohn, S. J., Carlson, J. S. \& Jensen, A. R. (1985). Speed of information processing in academically gifted youths. Personality and Individual Differences, 6(5), 621-629.

Crawford, J. R., Deary, I. J., Allan, K. M. \& Gustaffson, J. E. (1998). Evaluating competing models of the relationships between inspection time and psychometric intelligence. Intelligence, 26(1), 27-42.

Deary, I. J., Der, G. \& Ford, G. (2001). Reaction time and intelligence differences: A population-based cohort study. Intelligence, 29(6), 389-399.

Der, G. \& Deary, I. J. (2003). IQ, reaction time and the differentiation hypothesis. Intelligence, 31(5), 491-503.

Eysenck, H. J. (1985). Revolution in the theory and measurement of intelligence. Psychological Assessment, 1(1,2), 99-158.

Fink, A. \& Neubauer, A. C. (2001). Speed of information processing, psychometric intelligence and time estimation as an index of cognitive load. Personality and Individual Differences, 30(6), 1.009-1.021.

Fink, A. \& Neubauer, A. C. (2005). Individual differences in time estimation related to cognitive ability, speed of information processing and working memory. Intelligence, 33(1), 5-26.

Grudnik, J. L. \& Kranzler, J. H. (2001). Meta-analysis of the relation between intelligence and inspection time. Intelligence, 29(6), 523-535.

Hick, W. (1952). On the rate of gain information. Quarterly Journal of Experimental Psychology, 4(1), 11-46.

Horn, J. L. \& Noll, J. C. (1997). Human cognitive capabilities: GfGc theory. Em D. P. Flanagan, J. L. Genshaft \& P. L. Harrison (Orgs.), Contemporary intellectual assessment: Theories, tests, and issues (pp. 53-91). New York: Guilford Press.

Hulme, C. \& Turnbull, J. (1983). Intelligence and inspection time in normal and mentally retarded subjects. British Journal of Psychology, 74(3), 365-370.
Hunt, E. (1999). Intelligence and human resources: Past, present and future. Em P. L. Ackerman, P. C. Kyllonen \& R. D. Roberts (Orgs.), Learning and individual differences: Process, trait and content determinants (pp. 3-30). Washington, DC: American Psychological Association.

Hyman, R. (1953). Stimulus information as a determinant of reaction time. Journal of Experimental Psychology, 45, 188-196.

Irwin, R. J. (1984). Inspection time and its relation to intelligence. Intelligence, 8(1), 47-65.

Jensen, A. R. \& Munro, E. (1979). Reaction time, movement time and intelligence. Intelligence, 3(2), 121-126.

Jensen, A. R. (1979a). Reaction time and intelligence. Em M. P. Friedman, J. P. Das \& N. O'Connor (Orgs.), Intelligence and Learning (pp. 3-30). New York: Plenum Press.

Jensen, A. R. (1979b). G: Outmoded theory or unconquered frontier? Creative Science \& Technology, 2(1), 16-29.

Jensen, A. R. (1993). Why is reaction time correlated with psychometric $g$ ? Curent Directions in Psychological Science, 2(1), 53-56.

Jensen, A. R., Schafer, E. W. \& Crinella, F. M. (1981). Reaction time, evoked brain potentials and psychometric $\mathrm{g}$ in the severely retarded. Intelligence, 5(2), 179-197.

Kranzler, J. H. \& Jensen, A. R. (1989). Inspection time and intelligence: A meta-analysis. Intelligence, 13(4), 329-347.

Larson, G. E. \& Saccuzzo, D. P. (1986). Jensen's reaction time experiments: Another look. Intelligence, 10(3), 231-238.

Levine, G., Preddy, D. \& Thorndike, R. L. (1987). Speed of information processing and level of cognitive ability. Personality and Individual Differences, 8(5), 599-608.

Lindley, R. H., Wilson, S. M., Smith, W. R. \& Bathurst, K. (1995). Reaction time and IQ: Shape of the task complexity function. Personality and Individual Differences, 18(3), 339-345.

Longstreth, L. E. (1984). Jensen's reaction time investigation of intelligence: A critique. Intelligence, 8(2), 139-160.

Longstreth, L. E., Walsh, D. A., Alcorn, M. B. \& Szzeeszulski, P. A. (1986). Backward masking, IQ, SAT and reaction time: Interrelationships and theory. Personality and Individual Differences, 7(5), 643-651.

Necka, E. (1992). Cognitive analysis of intelligence: The significance of working memory processes. Personality and Individual Differences, 13(9), 1031-1046.

Nettelbeck, T. \& Kirby, N. H. (1977). The importance of structure to the acquisition of skill amongst the middly retarded. Travail Human, 40(1), 29-40.

Nettelbeck, T. \& Kirby, N. H. (1983a). Measures of timed performance and intelligence. Intelligence, 7(1), 39-52.

Nettelbeck, T. \& Kirby, N. H. (1983b). Retarded-nonretarded differences in speed of processing. Australian Journal of Psychology, 35(3), 445-453.

Nettelbeck, T. (1982). Inspection time: An index for intelligence. Quarterly Journal of Experimental Psychology: Human Experimental Psychology, 34(2), 299-312.

Nettelbeck, T. (2001). Correlation between inspection time and psychometric abilities. A personal interpretation. Intelligence, 29(6), 459-474.

Neubauer, A. C. (1991). Intelligence and RT: A modified Hick paradigm and a new RT paradigm. Intelligence, 15(2), 175192. 
O’Connor, T. A. \& Burns, N. R. (2003). Inspection time and general speed of processing. Personality and Individual Differences, 35(3), 713-724.

Osmon, D. C. \& Jackson, R. (2002). Inspection time and IQ fluid or perceptual aspects of intelligence? Intelligence, 30(2), 119127.

Petrill, S. A., Luo, D., Thompson, L. A. \& Detterman, D. K. (2001). Inspection time and the relationship among elementary cognitive tasks, general intelligence, and specific cognitive abilities. Intelligence, 29(6), 487-496.

Rinderman, H. \& Neubauer, A. C. (2004). Processing speed, intelligence, creativity, and school performance: Testing of causal hypothesis using structural equation models. Intelligence, 32(6), 573-589.

Schwartz, S., Griffin, T. M. \& Brown, J. (1983). Power and speed components of individual differences in letter matching. Intelligence, 7(4), 369-378.

Sen, A., Jensen, A. R., Sen, A. K. \& Arora, I. (1983). Correlation between reaction time and intelligence in psychometrically similar groups in America and India. Applied Research in Mental Retardation, 4(2), 139-152.

Sharp, S. (1898/99). Individual psychology: A study in psychological method. American Journal of Psychology, 10(3), 329-391.

Smith, G. A. (1986). Inspection time and response strategies in a choice response task. Personality and Individual Differences, 7(5), 701-708.

Smith, G. L. \& Stanley, G. (1983). Clocking g: Relating intelligence and measures of timed performance. Intelligence, 7(4), 353368.

Sternberg, S. (1966). High speed scanning in human memory. Science, 153(3736), 652-654.

Stough, C., Thompson, J. C., Bates, T. C. \& Nathan, P. J. (2001). Examining neurochemical determinants of inspection time. Development of a biological model. Intelligence, 29(6), 511522.
Vernon, P. A. \& Weese, S. E. (1993). Predicting intelligence with multiple speed of information-processing tests. Personality and Individual Differences, 14(3), 413-419.

Vernon, P. A. (1981). Reaction time and intelligence in mentally retarded. Intelligence, 5(4), 345-355.

Vernon, P. A. (1983a). Recent finding in the nature of g. Journal of Special Education, 17(3), 388-400.

Vernon, P. A. (1983b). Speed of information processing and general intelligence. Intelligence, 7(1), 53-70.

Vernon, P. A. (1986). Inspection time: Does it measure intelligence? Personality and Individual Differences, 7(5), 715-720.

Vernon, P. A., Nador, S. \& Kantor, L. (1985). Reaction time and speed of processing: Their relationships to timed and untimed measures of intelligence. Intelligence, 9(4), 357-374.

Vickers, D. \& Smith, P. L. (1986). The rationale for the inspection time index. Personality and Individual Differences, 7(5), 609624.

Vigneau, F., Blanchet, L., Loranger, M. \& Pépin, M. (2002). Response latencies measured on IQ tests: Dimensionality of speed indices and the relationship between speed and level. Personality and Individual Differences, 33(1), 165-182.

Williams, B. R., Hultshch, D. F., Strauss, E. H., Hunter, M. A. \& Tannock, R. (2005). Inconsistency in reaction time across the life span. Neuropsychology, 19(1), 88-96.

Wissler, C. (1901). The correlation of mental and physical tests. Psychological Monographs, 3(6), 1-62.

Zhengkui, L., Jiannong, S. \& Li, C. (2003). The relations between inspection time and intelligence of children. Acta Psychologica Sinica, 35(6), 823-829.

Recebido em 08.05.2004

Primeira decisão editorial em 04.02.2005

Versão final em 01.04.2005

Aceito em 06.04.2005 